\title{
PERCEÇÕES DOS PROFISSIONAIS DE RE- LAÇÕES PÚBLICAS SOBRE O USO ÉTICO DOS MEDIA SOCIAIS EM PORTUGAL E NO BRASIL
}

\author{
SÓNIA PEDRO SEBASTIÃO \\ UNIVERSIDADE DE LISBOA \\ LISBOA, PORTUGAL \\ SSEBASTIAO@ISCSP.ULISBOA.PT \\ GIOVANA ZULATO \\ UNIVERSIDADE DE LISBOA \\ LISBOA, PORTUGAL \\ GIOVANAZULATO@YAHOO.COM.BR \\ TÂNIA BELO SANTOS \\ UNIVERSIDADE DE LISBOA \\ LISBOA, PORTUGAL \\ TANIABELOSANTOS@GMAIL.COM
}




\section{PERCEÇÕES DOS PROFISSIONAIS DE RELAÇÕES PÚBLICAS SOBRE O USO ÉTICO DOS MEDIA SOCIAIS EM PORTUGAL E NO BRASIL}

Resumo: Baseado no método e no questionário, e inspirado pela teoria de Toledano e Avidar (2016), este artigo é focado no estudo das relações públicas digitais de um ponto de vista ético. O principal objetivo é aferir as atitudes dos profissionais de relações públicas (RP) relativamente ao uso profissional dos media sociais, identificando questões éticas e as implicações das suas perceções. Para isso, recorremos ao método quantitativo suportado num inquérito por questionário distribuído online. Este foi respondido por RP portugueses e brasileiros e os resultados não mostraram diferenças significativas entre as amostras respondentes destes dois contextos socioculturais. Quanto às dimensões éticas, verificou-se que a autenticidade e a transparência não são veementemente defendidas nos dois países.

Palavras-chave: Relações Públicas. Media Sociais. Ética. Inquérito por Questionário.

\section{INTRODUÇÃO}

A investigação no campo das relações públicas digitais tem aumentado nos últimos anos, verificando-se um aumento significativo de publicações científicas (Ye \& Ki, 2012; Duhé, 2015). No entanto, esta investigação não tem um sólido enquadramento teórico aplicado (as teorias da simetria e dialógicas ainda predominam), os estudos qualitativos em profundidade são escassos e existe uma considerável concentração de estudos no uso das plataformas digitais em detrimento da análise crítica dos efeitos e em formas de melhorar o uso do meio.

A maior parte dos estudos realizados até ao momento tende a providenciar recomendações aos profissionais sobre como poderão beneficiar com o uso destas tecnologias na construção de relações entre as organizações e os públicos, na resolução de problemas e no estabelecimento de objetivos socialmente responsáveis. A argumentação inclui a necessidade de adoção de comportamentos éticos (e.g. Brown, 2009; Macnamara \& Zerfass, 2012) considerada como uma das principais preocupações da pesquisa de relações públicas que inclui os media sociais (Duhé, 2015). Neste artigo, incluímos sob a denominação de media sociais os perfis em redes sociais online (como o facebook, instagram, twitter, entre outros), os canais em plataformas digitais de vídeo (como o youtube, o vimeo, entre outros) e os weblogues. Considerando a ausência de investigação reflexiva, este estudo pretende contribuir para o entendimento das relações públicas digitais de um ponto 
de vista ético, abordando a prática profissional das relações públicas em diferentes contextos socioculturais, em particular em contextos latinos. Não procuramos, contudo, discutir a ontologia da ética nem os traços do comportamento ético (para isso recomendamos a leitura do trabalho de Bowen, Hung-Baesecke, \& Chen, 2016), apenas procurar aferir a importância percebida deste tipo de comportamento quando os media sociais são usados na prática das relações públicas em Portugal e no Brasil.

A ética é, por conseguinte, abordada considerando especificamente a perceção sobre as práticas comunicativas previamente selecionadas por Toledano e Avidar (2016). Assim sendo, este artigo é iniciado por uma revisão de literatura sobre a pesquisa de relações públicas digitais e ética; segue-se a caracterização dos contextos socioculturais e profissionais de Portugal e Brasil. Apesar dos países considerados terem uma matriz lusófona comum, existem diferenças históricas, geográficas e traços culturais que os levam a diferentes desempenhos em índices internacionais (Index of Human Freedom, The Reporters without Borders' World Press Freedom Index, Corruption Perceptions Index) que poderão influenciar a perceção sobre práticas profissionais comunicativas éticas. Os resultados são posteriormente apresentados e discutidos procurando analisar a possível influência destes contextos nas perceções dos profissionais inquiridos sobre práticas comunicativas digitais eticamente aceitáveis ou não.

\section{MEDIA SOCIAIS E RELAÇÕES PÚBLICAS}

A integração dos media sociais em estratégias de comunicação organizacional tem sido intensivamente discutida nos últimos anos. A investigação sobre media sociais é mesmo uma das mais prolíficas em estudos de comunicação apesar da limitação do seu escopo teórico (Osch \& Coursaris, 2014 e 2015). O foco da pesquisa tem alternado de uma perspetiva normativa relacionada com abordagens teóricas ligadas à simetria e ao diálogo (e.g. Kent \& Taylor, 1998; Wright, 2001; Kent, Taylor, \& White, 2003; Brown, 2009, Kaplan \& Haenlein, 2010; Duhé, 2012), para a argumentação sobre os benefícios, limites e pré-requisitos estruturais das estratégias digitais (e.g. Kietzmann, Hermkens, McCarthy \& Silvestre, 2011; Treem \& Leonardi, 2012; Macnamara \& Zerfass, 2012) e a realização de estudos empíricos sobre práticas digitais (e.g. Kim, Park \& Wertz, 2010; Gilpin, 2010; Men \& Tsai, 2012; Einwiller \& Steilen, 2015). 
Apesar da investigação científica (e.g. Diga \& Kelleher, 2009; Wright \& Hinson, 2015) e de estudos globais (tais como: o European Communication Professionals Skills and Innovation Programme - ECOPSI - e o European Communication Monitor - ECM) indicarem que os profissionais de comunicação e relações públicas demonstram conhecimento sobre os media sociais e os seus princípios, as organizações tendem a não usá-los explorando de forma eficiente e efetiva o seu potencial simétrico e dialógico. Desta forma, faIham o uso da comunicação na sua essência "mais ética", atendendo a que o diálogo permite o equilíbrio de poder entre os intervenientes no processo comunicativo possibilitando discernir a falsidade e fomentar a honestidade (Kent \& Taylor, 2002, p. 22). Existem dificuldades no envolvimento dialógico, na mutualidade de compromisso e interesses, uma vez que as organizações têm fins predeterminados que podem por em causa a continuidade, a confiança e a autenticidade das relações com os públicos. Os media sociais falham, por conseguinte, a sua "promessa dialógica" (McAllister-Spooner, 2009), atendendo a que são, acima de tudo, sistema tecno-sociais: os instrumentos tecnológicos permitem e constrangem o desenvolvimento de atividades sociais que criam conhecimento que, por sua vez, será produzido, difundido e consumido com o auxílio dos instrumentos tecnológicos (Fuchs, 2014, p. 37).

Nos últimos anos, tem sido constatado que, tecnicamente, os media sociais oferecem várias oportunidades de interação com os utilizadores da internet, servindo de suporte à comunicação, à partilha de conteúdos e ao diálogo, contudo, não são automaticamente dialógicos uma vez que as organizações podem não ter interesse na mutualidade, na empatia ou no compromisso (Kent \& Taylor, 2002). Os media sociais podem promover conversas rápidas e instantâneas (Goldstraw, 2015), mas nem todas as organizações estão preparadas (ou desejam) encetar essas conversas, ou sequer usar os media sociais no contacto com os seus públicos. Existem organizações que não têm recursos ou estruturas adequados para se envolver; outras não têm cultura comunicativa ou o diálogo pode não ser a forma de comunicação mais adequada à sua natureza e missão (Young, 2015). Finalmente, o diálogo comporta riscos que podem ser nefastos para certas organizações.

Em síntese, a missão e os constrangimentos das organizações devem ser considerados antes da tomada de decisão de usar os media sociais numa estratégia de comunicação. A sua utilização deve ser integrada e adequada à cultura organizacional. É contudo importante ter em consideração que 
os media sociais, quando incluídos na comunicação das organizações, devem integrar a sua estratégia e não ser usados de forma isolada (Goldstraw, 2015, p. 355). Além disso, e podendo a organização não definir perfis nos media sociais, não pode ignorar a sua existência e deverá promover a monitorização dos mesmos. A monitorização sistemática dos media sociais é obrigatória atendendo ao risco que colocam na gestão da reputação e identidade organizacionais (Motion, Heath \& Leitch, 2016, p. 36). A organização pode decidir não usar os media sociais, contudo não pode decidir se os seus públicos os vão usar e se nos mesmos a vão mencionar (de modo positivo ou negativo) podendo as mensagens produzidas e partilhadas afetar a reputação organizacional.

O potencial e a forma com os media sociais têm sido utilizados no campo da comunicação e na prática das relações públicas têm captado a atenção de vários académicos com maior ou menor otimismo (Valentini, 2015). Apesar do criticismo anteriormente apresentado, um estudo recente desenvolvido com PR israelitas destaca que, desde 2010 e com a crise económica dos media tradicionais, a blogosfera tem sido usada por estes profissionais como forma de divulgação e promoção de assuntos em áreas específicas como: lifestyle, entretenimento, cosmética, turismo e cultura. Os autores destes blogues estão disponíveis para colaborar com os profissionais de relações públicas em troca de compensações financeiras e sociais, abdicando da sua independência e aceitando o controlo dos PR sobre o conteúdo dos seus blogues (Lahav \& Roth-Cohen, 2016). Comparando este estudo com os resultados de Kent (2008) e seguindo as premissas da teoria da difusão de inovações (Rogers, 1962/2003), PR e bloggers podem, atualmente, estar recetivos às alterações provocadas pelos blogues na prática profissional. Contudo, esta aceitação provoca preocupações éticas que ainda não foram discutidas.

\section{MEDIA SOCIAIS E ÉTICA}

Como observado anteriormente, os media sociais são maioritariamente estudados no contexto dos seus usos (por exemplo, nas relações com os órgãos de comunicação social e com os jornalistas) e na aferição do seu potencial dialógico. As questões éticas permanecem ausentes da maior parte dos estudos analisados (Vercic, Vercic, \& Sriramesh, 2014; Duhé, 2015). Algumas exceções são: a discussão sobre o uso ético dos media sociais pelos 
RP desenvolvido com profissionais neozelandeses e israelitas por Toledano e Wolland (2011) e por Toledano e Avidar (2016); os estudos de caso e a sistematização de princípios éticos proposta por Bowen (2013 e 2013b) e o trabalho desta autora com colegas sobre a importância da ética como percussora da construção de relações entre as organizações e os seus públicos (Bowen at al., 2016). Finalmente, Valentini (2015) enfatiza criticamente que devido "à poluição" do online com conteúdos falsos e práticas não éticas, os públicos tendem a imunizar-se a mensagens persuasivas veiculadas nos media sociais. Consequentemente compete ao profissional de relações públicas avaliar a natureza das práticas da organização em torno do uso destes instrumentos. Só assim poderá providenciar conselhos e sensibilizar a direção e os colegas para a importância e os efeitos de práticas comunicativas éticas (Valentini, 2015, p. 175).

Todavia a profissão de relações públicas não está isenta de desafios éticos. Como sumariado por L'Étang (2011, p. 221), pontos-chave no debate sobre a conduta destes profissionais incluem: manipulação, mentira, omissões, subornos, distorção dos canais de comunicação ao serviço dos poderosos, gerando falta de autenticidade e impondo discursos promocionais. Como podem, então, estes profissionais servir de "guardiões éticos" ou denunciadores de condutas pouco éticas das organizações?

Atendendo à natureza da sua prática - que visa a construção de opinião pública - há interligação dos conceitos de relações públicas e ética desde as origens da profissão. Autores como Cheney, Munshi, May e Ortiz (2011) referem que a teoria da excelência defende que a ética perpassa a definição da prática, seguindo o pensamento ético com origens em Platão. Por exemplo, o modelo simétrico bidirecional evita o problema do relativismo ético atendendo a que a ética é definida como um processo das relações públicas e não como um resultado (Grunig \& Grunig, 1992, p. 308). Ao assumirem que as relações com os públicos podem ser usadas para equilibrar e tornar os processos de decisão organizacionais mais simétricos, a concretização de objetivos de longo prazo integra inputs dos públicos. Desta forma, fomentam-se relações mais benéficas e duradouras - e inerentemente mais éticas - entre a organização e os seus públicos (Bowen, 2007). No entanto, é necessário reter que a ética é sobre "fazer a coisa certa em dada situação" (Cheney et al., 2011, p. 3), observando um enquadramento filosófico, permitindo interpretações plurais e práticas que refletem e incentivam diferenças de ambientes culturais específicos (Ess, 2011, p. 217). A ética é uma disciplina 
que aborda o bem e o mal impregnados por dever e obrigação moral (Hyde, 2011, p. 32).

A rápida mudança provocada pela evolução tecnológica, a emergência da internet e a disseminação dos media sociais reavivam antigas questões éticas e colocam novos desafios aos RP (Phillips \& Young, 2009; Jensen, 2011). Contudo, estes novos desafios ainda estão pouco explorados pelos investigadores de relações públicas. As organizações necessitam de ser aceites pelos seus públicos como legítimas e responsáveis (Toledano \& Wolland, 2011, p. 44), sendo a legitimidade baseada na perceção dos públicos construída a partir das práticas comunicativas das organizações. Se as atividades de comunicação e relações públicas não forem desempenhadas de forma ética, podem surgir crises de legitimidade que comprometem a sobrevivência das organizações. É importante não esquecer que a prática das relações públicas tem uma reputação negativa por comportamentos manipulativos não éticos (Moloney, 2000; L’Étang, 2011) associados à sua história e à sua tradição retórica (Heath, 2006; Valentini, 2015).

O comportamento ético é percursor da construção de relações autênticas, de longo prazo com os públicos e, por conseguinte, é beneficiador da efetividade e reputação das organizações (Bowen et al., 2016, p. 1), sendo condição necessária às relações de confiança. O comportamento ético e todos os esforços de comunicação desenvolvidos pelas organizações visam influenciar a forma como a reputação organizacional é percebida pelos públicos. Estes esforços incluem a presença no ambiente digital e a forma como a organização se apresenta nos media sociais (Gilpin, 2010, p. 265).

Na prática das relações públicas, o comportamento ético inclui: o direito de privacidade; acordos de confidencialidade; consentimento informado; credibilidade e veracidade de informação; mutualidade (ou reciprocidade) e transparência (Goldstraw, 2015). Estes princípios não se coadunam com ações comunicativas não éticas existentes em ambiente digital, tais como: falsos perfis em media sociais; websites de ataque (criados por grupos ou cidadãos anónimos para parodiar, denegrir e denunciar organizações, ou mesmo para ações de marketing de guerrilha); chain letters (emails enviados em cadeia destinados a divulgar ideias, fazer denúncias, recolher assinaturas, gerar tráfego, disseminar informações falsas e rumores) e phishing (também associado a emails mas sem a natureza viral das chain letters, tem como objetivo recolher dados pessoais e códigos de acesso a serviços online). 
Reforçando as dimensões de uma prática profissional ética, sublinhamos os princípios de Jensen (2011). Este autor advoga pela transparência, veracidade, autenticidade e respeito pelo mercado (quer pelos concorrentes do lado da oferta, quer pelos consumidores e clientes do lado da procura) enfatizando que quem está presente online e usa estas plataformas para comunicar deve informar sobre os pagamentos e ofertas que recebe para produzir conteúdos e não deve plagiar ou omitir as suas afiliações.

Com base na filosofia de Kant, Bowen (2013) sintetizou o comportamento ético a adotar nos media sociais nos seguintes princípios: justiça; prudência; transparência; autenticidade; racionalidade; clareza; veracidade; consistência; e evitar do engano e do segredo. Para a autora, a fragmentação e mudança rápida do ambiente comunicativo aumenta as exigências éticas, uma vez que os públicos estão presentes online e exigem cada vez maior simetria comunicativa e relacional. Assim sendo, é fundamental que os RP compreendam as regras dos media sociais de modo a incentivarem o envolvimento e evitarem mensagens e retórica promocionais (Motion et al., 2016, p. 32).

Em síntese, e atendendo às dimensões/eixos éticos apresentados por Jensen (2011), Bowen (2013) e Goldstraw (2015), este artigo destaca: a transparência, associada à informação da identidade, interesses e benefícios subjacentes às mensagens; veracidade da informação e da prática; autenticidade das vozes (autores) evitando o engano; e o respeito pelos públicos e pelo mercado.

\section{CONTEXTOS SOCIOCULTURAIS DE PORTUGAL E BRASIL}

Os profissionais de relações públicas utilizam diferentes instrumentos de comunicação para disseminar as suas mensagens e cumprir a sua missão profissional. Apesar da existência de um modelo global de prática, as funções destes profissionais são determinadas por contextos culturais (L'Étang, 2011). A utilização de instrumentos e meios de comunicação ocorre em contextos democráticos, de liberdade e transparência, como observado por Toledano e Avidar (2016), cujo modelo analítico é seguido neste artigo. É importante, por conseguinte, atentar nos índices internacionais e nos desempenhos dos dois países em estudo: Portugal e Brasil.

No Index of Human Freedom (Fraser Institute, 2013) que inclui a liberdade de movimento, a liberdade de expressão (incluindo a liberdade de imprensa) e a liberdade de associação, Portugal encontra-se em \#25 e o Brasil 
em \#50, num total de 123 países (Vásquez \& Stumberger, 2012).

Através do Corruption Perceptions Index (2014), o ranking da Transparency International mede os níveis percebidos por um conjunto de especialistas sobre a corrupção no setor público de 175 países e territórios. O desempenho de um país será melhor com governos "abertos" onde os cidadãos podem responsabilizar os líderes. Em sentido inverso, o mau desempenho de um país indica a prevalência de subornos, a falta de punição para quem pratica atos corruptos e a existência de serviços públicos que não colmatam as necessidades dos cidadãos. Portugal surge na 31. ${ }^{\text {a }}$ posição deste ranking evidenciando que detém níveis médios de corrupção no contexto ocidental, enquanto o Brasil surge na 69. a posição. Este país tem, nos últimos anos, sido palco de vários escândalos de corrupção envolvendo empresas públicas e membros do governo.

De acordo com o World Press Freedom Index (2015) dos Reporters Without Borders, Portugal surge no \#26 numa lista de 180 países, ascendendo do \#30 de 2014, assinalando a inexistência de violência contra jornalistas e de abusos de imprensa. Graças à liberdade de expressão, ao pluralismo e aos índices de corrupção, a profissão de relações públicas desenvolveu-se no país em dois eixos principais: por um lado, as exigências éticas relacionadas com os respetivos códigos de conduta; por outro lado, com criticismo público e conotações com propaganda e manipulação da opinião pública (Gonçalves, 2012).

A situação no Brasil é diferente. O Brasil surge no \#99 em termos de segurança dos jornalistas e de concentração das empresas de media nas mãos de poucos empresários. Atos de violência contra jornalistas e assistentes são frequentes e, em 2014, dois perderam a vida na cobertura dos protestos cívicos. A profissão de jornalista é, ainda, dominada e limitada por poderes económicos e políticos.

Em suma, Portugal apresenta melhores desempenhos que o Brasil nos índices internacionais considerados. No Index of Human Freedom a diferença é de 25 pontos, numa lista de 123 países; no Corruption Perceptions Index a diferença é de 38 pontos, em 175 países. O fosso torna-se mais significativo no World Press Freedom Index, estando o Brasil 73 pontos abaixo do desempenho de Portugal (em 180 países).

Como os países em estudo apresentam diferentes níveis de transparência e liberdades humanas, as profissões ligadas à comunicação - como o jornalismo e as relações públicas - são exercidas em contexto socioculturais 
diferentes. Afetarão estes contextos as perceções éticas dos profissionais de relações públicas sobre o uso dos media sociais? Poderão contextos mais respeitadores das liberdades humanas inspirar atitudes profissionais mais éticas?

\section{O SETOR dAs RELAÇÕES PÚBLICAS E O USO DA INTERNET EM PORTUGAL E NO BRASIL}

Existem, em Portugal, três associações profissionais que competem na representação do setor: a Associação Portuguesa de Agências de Publicidade, Comunicação e Marketing (APAP), criada em 1969 e inicialmente representante de Agências de Publicidade, tendo recentemente alargado o seu escopo de atuação; a Associação Portuguesa de Comunicação Empresarial (APCE), fundada em 1990 e que junta profissionais, empresas, académicos e estudantes; e a Associação Portuguesa de Empresas de Comunicação e Relações Públicas (APECOM) cujo principal objetivo é a representação dos seus membros e assegurar a dignidade da profissão de consultoria em comunicação e relações públicas, seguindo os princípios do Código de Estocolmo da International Communication Consultancy Organization (ICCO).

O diretório das empresas do setor indica a existência de aproximadamente 180 consultoras de comunicação e relações públicas, existindo elevada fragmentação da prática profissional e da perceção do mercado sobre os serviços providenciados. Esta situação é motivada essencialmente por dois fatores: a falta de associativismo e de uma atitude colaborativa entre as empresas (Sebastião \& Azevedo, 2014) e a dimensão das Consultoras que são maioritariamente micro (freelancers em nome individual e empresas com menos de 10 colaboradores), com algumas de pequena e média dimensão.

A ausência de uniformidade de designações, o constante criar de rótulos para funções e responsabilidades, e a dispersão de atividades desenvolvidas por estes profissionais também contribui para uma perceção menos correta ou positiva da profissão (Gonçalves, 2007). Por um lado, existem várias subáreas de atuação da sua responsabilidade, por exemplo: as relações com os media; o apoio ao marketing; a comunicação interna; as relações institucionais e governamentais; a organização de eventos sociais e culturais. Por outro lado, nos países latinos a expressão "relações públicas" é uma expressão lata associada ao atendimento ou vendas em loja, provocando a adoção de outras designações como: Comunicação Organizacional, Institucional, ou 
mesmo, Corporativa.

A Associação Brasileira de Agências de Comunicação (ABRACOM) é a representante no país das empresas que prestam serviços de comunicação e relações públicas. De acordo com informação no website da associação, o setor terá sido responsável por faturações superiores a dois biliões de reais em 2014 e envolve cerca de catorze mil profissionais com várias formações, que trabalham em equipas multidisciplinares. A Lei Federal 5.377 (1967), regulada pelo Decreto 63.283 (1968), determina que estes profissionais devem ter formação académica superior em relações públicas e o seu registo enquanto tal (creditação). Contudo, a prática é diferente atendendo a que o mercado brasileiro se desenvolveu à margem da lei. A organização desenvolve inclusivamente uma campanha defendendo a necessidade de múltiplas formações para enriquecer o setor e cujo slogan é: "somos todos comunicação", envolvendo jornalistas e RP. O Conselho Federal dos Profissionais de Relações Públicas (CONFERP) discorda desta posição alegando que o exercício de relações com os media por profissionais sem formação académica superior em relações públicas, e especialmente por jornalistas, é uma prática ilegal e não ética.

A ABRACOM é afiliada da ICCO mas tem o seu código ético próprio disponível online (ABRACOM, s/d). Reúne 205 membros, catorze sem website, 54 sem oferta de serviços de comunicação digital. 137 agências oferecem estes serviços com diferentes nomenclaturas, onde identificamos: comunicação digital; planeamento, atualização e monitorização de media sociais; soluções digitais; gestão de redes sociais, entre outras. Este cenário revela que as agências de comunicação brasileiras reconhecem a importância de prestar serviços em media sociais.

Quanto ao uso da internet, em Portugal, existe cerca de 67,6\% de penetração (Internet World Stats, 2015), com os portugueses a revelarem elevados índices de utilização de media sociais e plataformas digitais, assim como, concordância com a utilização dos media sociais pelas marcas para fins de interação com os seus consumidores. Em conformidade, cerca de $69 \%$ dos utilizadores dos media sociais revelam seguir marcas nestas redes (Marktest, 2015).

No Brasil, a penetração da internet ainda não chega a 50\% da população, tendo contudo evoluído a percentagem dos brasileiros que usam a internet diariamente de 26\% em 2014 para 37\% em 2015 (Pesquisa Brasileira de Mídia, 2015). Já a GlobalWebIndex (2014) revela que os media sociais têm ocupado 
cada vez mais tempo dos brasileiros. Não obstante a evolução positiva, cerca de $51 \%$ dos brasileiros ainda não tem acesso à internet, o que é resultado de exclusões económicas, demográficas e educacionais.

\section{NOTA METODOLÓGICA}

Com o objetivo de aferir as atitudes dos profissionais de relações públicas (RP) relativamente ao uso profissional dos media sociais identificando questões éticas e as implicações das suas perceções, foi desenvolvido um estudo quantitativo baseado na aplicação de um inquérito por questionário disponibilizado numa plataforma digital (Survey Monkey) cuja hiperligação foi enviada a profissionais de relações públicas que usam os media sociais regularmente. Este pré-requisito foi assegurado com a inserção de uma pergunta filtro no questionário e o envio da hiperligação foi segmentado através do contacto com profissionais de empresas de Consultoria de Comunicação e Relações Públicas com serviços de comunicação digital, em Portugal e no Brasil. Foi ainda solicitado aos respondentes que fizessem chegar a hiperligação a colegas de profissão. As respostas ao questionário, aplicado a amostras de conveniência construídas em bola de neve junto de RP portugueses e brasileiros, foram recolhidas nos meses de novembro e dezembro de 2015.

Além da questão filtro, o questionário incluiu cinco questões de caracterização sociodemográfica dos inquiridos e treze afirmações sobre práticas profissionais mais e menos éticas, definidas por Toledano e Avidar (2016), com o intuito de aferir atitudes sobre a prática normativa, a transparência, a autenticidade e o respeito pelo mercado.

Apesar do pedido de colaboração endereçado às associações de profissionais de ambos os países, verificamos que os profissionais se mostraram relutantes em colaborar. Falta de tempo, desconsideração pela importância da investigação, falta de vontade de colaborar com estudos académicos e a delicadeza do tema da ética, diretamente associado à conduta profissional, são algumas das explicações que podemos avançar para a dimensão das amostras.

Apesar de todas as dificuldades, recolheram-se 100 respostas de profissionais portugueses (NPT=100) e 44 de profissionais brasileiros $(N B=44)$. As duas amostras apresentam características sociodemográficas similares. Em síntese, ambas são maioritariamente femininas; os respondentes têm 
uma idade média localizada entre os 30 e os 35 anos e têm cursos de ensino superior. Os inquiridos trabalham essencialmente no setor privado e a sua experiência profissional varia entre os 4 e os 20 anos.

Nenhuma das amostras pode ser considerada representativa do setor da consultoria de relações públicas atendendo ao processo de amostragem e dimensão da amostra. Não obstante, os resultados apresentam dados interessantes sobre a opinião dos profissionais em relação a práticas de comunicação digital, relacionadas com aspetos éticos e alguns insights sobre o uso dos media sociais pelos RP portugueses e brasileiros.

Considerando a revisão de literatura anteriormente apresentada formularam-se as seguintes questões de pesquisa:

QP1. Afetarão diferentes contextos socioculturais a atitude do RP em relação a aspetos éticos no uso dos media sociais?

QP2. Qual das dimensões éticas suscita maior divisão nas respostas: a transparência, a autenticidade, a veracidade ou o respeito pelo mercado?

As respostas às treze afirmações apresentadas aos RP foram organizadas considerando quatro eixos éticos inspirados nos modelos de Jensen (2011), Bowen (2013) e Goldstraw (2015): a prática normativa; a transparência; a autenticidade; a veracidade e o respeito pelo mercado. Os inquiridos declararam a sua atitude em relação às afirmações usando uma escala de Likert (1. Discordo Totalmente; 2. Discordo; 3. Não concordo nem discordo; 4. Concordo; 5. Concordo Totalmente). Os resultados são sintetizados considerando a média $(\mu)$ e o desvio padrão (DP) das respostas, assim como, um teste $T$ de duas amostras com variâncias desiguais, procurando evidenciar a significância das diferenças de resposta entre as duas amostras.

\section{RESULTADOS}

Baseado eticamente, o eixo da prática normativa visa evidenciar se a componente retórica das relações públicas - e a sua natureza de certa forma dissimulada (Valentini, 2015) - é percebida e aprovada pelos profissionais de relações públicas inquiridos. Considerando as respostas ao inquérito (tabela 1), é possível observar que os inquiridos brasileiros se sentem ligeiramente mais treinados para lidar com questões éticas relacionadas com a comunicação organizacional utilizando os media sociais, o que pode estar relacionado com a exigência legal brasileira em termos de formação académica destes profissionais. 
As duas amostras inquiridas consideram que os RP são responsáveis pela conduta ética da organização nos media sociais devendo, por isso, formar e guiar os restantes recursos humanos da organização (incluindo a direção), assumindo-se como "guardiões éticos" (L’Étang, 2004). Verificamos também grande concordância com a necessidade das organizações publicarem uma política comunicativa/manual para orientação da sua comunicação nos media sociais. Estas políticas permitem estabelecer mecanismos de governança para a publicação de conteúdos, accountability e responsabilização, o que, de certa forma, normalizaria a presença digital da organização (Motion et al., 2016, p. 25).

Tabela 01: Resultados sobre a prática normativa

\begin{tabular}{|c|c|c|c|c|c|c|c|}
\hline Afirmações & Brasil & Brasil & Brasil & $\begin{array}{c}\text { Portu- } \\
\text { gal }\end{array}$ & $\begin{array}{l}\text { Portu- } \\
\text { gal }\end{array}$ & $\begin{array}{c}\text { Portu- } \\
\text { gal }\end{array}$ & Teste $\mathrm{T}$ \\
\hline & $\mathrm{Nb}$ & $\mu$ & DP & Npt & $\mu$ & DP & \\
\hline $\begin{array}{l}\text { Considero-me for- } \\
\text { mado para lidar com } \\
\text { questões éticas rel- } \\
\text { acionadas com a } \\
\text { comunicação orga- } \\
\text { nizacional utilizando } \\
\text { plataformas digitais } \\
\text { (media sociais). }\end{array}$ & 44 & 4,2 & 0,82 & 100 & 3,99 & 0,92 & $\begin{array}{c}t(91)=1,99, \\
p<0,17\end{array}$ \\
\hline $\begin{array}{l}\text { Os profissionais } \\
\text { de comunicação e } \\
\text { relações públicas } \\
\text { deve ter a respons- } \\
\text { abilidade pela con- } \\
\text { duta ética da orga- } \\
\text { nização nos media } \\
\text { sociais, formar e } \\
\text { guiar os colabora- } \\
\text { dores da organi- } \\
\text { zação, incluindo a } \\
\text { direção e as chefias. }\end{array}$ & 44 & 4,5 & 0,59 & 100 & 4,43 & 0,83 & $\begin{array}{c}t(113)=1,98, \\
p<0,57\end{array}$ \\
\hline
\end{tabular}




\begin{tabular}{|c|c|c|c|c|c|c|c|}
\hline $\begin{array}{l}\text { Os media sociais } \\
\text { melhoraram o con- } \\
\text { trolo dos profission- } \\
\text { ais de comunicação } \\
\text { e relações públicas } \\
\text { sobre a distribuição } \\
\text { das mensagens } \\
\text { sobre a(s) organi- } \\
\text { zação(ões) em que } \\
\text { prestam serviço. }\end{array}$ & 44 & 3,41 & 1,11 & 100 & 3,29 & 1,23 & $\begin{array}{c}t(91)=1,99, \\
p<0,57\end{array}$ \\
\hline $\begin{array}{l}\text { Os media sociais per- } \\
\text { mitem ao profission- } \\
\text { al de comunicação } \\
\text { e relações públicas } \\
\text { elevar o seu estatu- } \\
\text { to organizacional e } \\
\text { inspirar a direção em } \\
\text { decisões mais social- } \\
\text { mente responsáveis } \\
\text { e éticas. }\end{array}$ & 44 & 3,57 & 0,87 & 100 & 3,69 & 0,85 & $\begin{array}{c}t(80)=1,99, \\
p<0,44\end{array}$ \\
\hline $\begin{array}{l}\text { Cada organização } \\
\text { deveria publicar uma } \\
\text { política comunicati- } \\
\text { va (ou manual) para } \\
\text { instruir os seus co- } \\
\text { laboradores na uti- } \\
\text { lização dos media } \\
\text { sociais. }\end{array}$ & 44 & 4,52 & 0,82 & 100 & 4,64 & 0,75 & $\begin{array}{c}t(76)=1,99, \\
p<0,42\end{array}$ \\
\hline
\end{tabular}

As duas amostras têm tendência para concordar que os media sociais são uma oportunidade para os RP elevarem o seu estatuto organizacional e inspirar a direção em decisões mais socialmente responsáveis e éticas. Enquanto a maioria dos respondentes brasileiros consideram que os media sociais melhoraram o controlo dos profissionais sobre a distribuição das mensagens relativas às organizações que servem; os respondentes portugueses têm opiniões divididas. Contudo, o resultado estatístico do Teste T entre amostras com variâncias desiguais mostra a não existência de diferenças significativas $[t(91)=1,99, p<0,57]$.

Comparando estes resultados com os de Toledano e Avidar (2016), as perceções dos RP portugueses aparentam ser mais próximas das perceções mais éticas dos profissionais neozelandeses que também revelaram opiniões divididas sobre o controlo das mensagens distribuídas recorrendo aos media sociais.

Quanto às afirmações enquadradas pelo eixo da transparência (tabela 
2), por um lado, os respondentes concordam que devem denunciar práticas de corrupção na organização dando primazia ao interesse público. Por outro lado, fornecer informação sobre conteúdos pagos, recompensas e patrocínios é considerado obrigatório pelas amostras inquiridas na prática das relações públicas digitais. Escrever comentários nos media sociais sem revelar a identidade ou pagamentos são práticas não éticas reconhecidas e rejeitadas pelos nossos inquiridos e pelos inquiridos de Toledano e Avidar (2016). Coerentemente utilizar um disclaimer (ferramenta ética onde é feita atribuição de autorias e interesses) é algo defendido pelos respondentes.

Tabela 02: Resultados sobre transparência

\begin{tabular}{|c|c|c|c|c|c|c|c|}
\hline Afirmações & Brasil & Brasil & Brasil & $\begin{array}{c}\text { Portu- } \\
\text { gal }\end{array}$ & $\begin{array}{l}\text { Portu- } \\
\text { gal }\end{array}$ & $\begin{array}{c}\text { Portu- } \\
\text { gal }\end{array}$ & Teste T \\
\hline & $\mathrm{Nb}$ & $\mu$ & DP & Npt & $\mu$ & DP & \\
\hline $\begin{array}{l}\text { O RP deve denunciar } \\
\text { práticas corrupção } \\
\text { na organização em } \\
\text { que trabalha dando } \\
\text { primazia ao interes- } \\
\text { se público. }\end{array}$ & 44 & 3,75 & 1,16 & 100 & 4,11 & 1,05 & $\begin{array}{c}t(75)=1,99, \\
p<0,09\end{array}$ \\
\hline $\begin{array}{l}\text { Não há problema } \\
\text { se o RP escrever co- } \\
\text { mentários nos me- } \\
\text { dia sociais sem rev- } \\
\text { elar quem lhe pagou } \\
\text { para o fazer. }\end{array}$ & 44 & 2,09 & 0,96 & 100 & 2,16 & 1,15 & $\begin{array}{c}t(98)=1,99, \\
p<0,71\end{array}$ \\
\hline $\begin{array}{l}\text { Boas práticas re- } \\
\text { querem RP, blog- } \\
\text { gers e outros espe- } \\
\text { cialistas de media } \\
\text { sociais revelem se } \\
\text { estão a ser pagos ou } \\
\text { agraciados por uma } \\
\text { organização para } \\
\text { produzirem e divul- } \\
\text { garem determinada } \\
\text { mensagem. }\end{array}$ & 44 & 4 & 0,96 & 100 & 4,01 & 1,08 & $\begin{array}{c}t(92)=1,99, \\
p>0,91\end{array}$ \\
\hline
\end{tabular}




\begin{tabular}{|l|l|l|l|l|l|l|l|}
\hline $\begin{array}{l}\text { Criaria um grupo } \\
\text { ativista para apoiar } \\
\text { o interesse do meu } \\
\text { empregador ou cli- } \\
\text { ente e pagar-lhe-ia } \\
\text { para publicarem } \\
\text { "a nossa versão da } \\
\text { história" nos media } \\
\text { sociais. }\end{array}$ & & 2,14 & 1,11 & 100 & 2,19 & 1,08 & $\begin{array}{c}\mathrm{t}(80)=1,99, \\
\mathrm{p}>0,75\end{array}$ \\
\hline
\end{tabular}

Existem opiniões divididas em relação à criação de grupos ativistas para divulgar histórias convenientes a uma organização. Os RP portugueses inquiridos tendem a discordar com esta prática não ética, mas um número significativo não tem opinião sobre o assunto. Existem, também, respondentes brasileiros que têm uma perceção positiva sobre esta prática. Uma vez mais, o resultado do Teste $T$ realizado indica a inexistência de diferenças significativas nas respostas das duas amostras inquiridas $[t(80)=1,99, p>0,75]$.

Comparando com os resultados de Toledano e Avidar (2016), as respostas recolhidas aproximam-se mais das atitudes menos éticas defendidas pelos RP israelitas, uma vez que os RP neozelandeses repudiaram veementemente esta prática não ética.

A autenticidade aparenta ser a dimensão ética que origina maior divisão de respostas (QP2). Um número significativo de RP portugueses considera difícil escrever blogues em nome dos líderes organizacionais e tendem a discordar com a prática não ética de escrever conteúdos sem se identificarem.

Os respondentes brasileiros não consideram difícil escrever em nome dos líderes organizacionais, evidenciando que a autenticidade das vozes nos media sociais não é algo importante. Como também observam Toledano e Avidar (2016, p. 166) escrever em blogues em nome de terceiros parece ter-se tornado prática comum. Da mesma forma, os inquiridos brasileiros tendem a discordar ou a não ter opinião sobre a prática não ética do anonimato ou do comentário online dissimulado.

Apesar das diferenças nos resultados sobre a autenticidade, os Testes T realizados voltam a não indicar diferenças significativas nas respostas recoIhidas junto dos inquiridos portugueses e brasileiros (ver tabela 3).

Tabela 03: Resultados sobre a autenticidade

\begin{tabular}{|c|c|c|c|c|c|c|c|}
\hline Afirmações & Brasil & Brasil & Brasil & $\begin{array}{c}\text { Portu- } \\
\text { gal }\end{array}$ & $\begin{array}{c}\text { Portu- } \\
\text { gal }\end{array}$ & $\begin{array}{c}\text { Portu- } \\
\text { gal }\end{array}$ & Teste T \\
\hline
\end{tabular}




\begin{tabular}{|l|c|c|c|c|c|c|c|}
\hline & Nb & $\boldsymbol{\mu}$ & DP & Npt & $\boldsymbol{\mu}$ & $\mathbf{D P}$ & \\
\hline $\begin{array}{l}\text { Para mim é difícil } \\
\text { escrever blogues } \\
\text { em nome do Presi- } \\
\text { dente/Diretor da or- } \\
\text { ganização uma vez } \\
\text { que os media sociais } \\
\text { requerem vozes/dis- } \\
\text { cursos autênticos. }\end{array}$ & 44 & 2,41 & 1,21 & 100 & 3,12 & 1,26 & $\begin{array}{c}\mathrm{t}(85)=1,99, \\
\mathrm{p}>0\end{array}$ \\
\hline $\begin{array}{l}\text { Não há problema se } \\
\text { os RP escreverem } \\
\text { comentários nos } \\
\text { media sociais sem } \\
\text { divulgarem a sua } \\
\text { identidade e/ou afil- } \\
\text { iação. }\end{array}$ & 44 & 2,5 & 1,05 & 100 & 2,29 & 1,13 & $\begin{array}{c}\mathrm{t}(88)=1,99, \\
\mathrm{p}<0,3\end{array}$ \\
\hline
\end{tabular}

Quanto à perceção das práticas relacionadas com a veracidade e o respeito pelo mercado (ver tabela 4), os respondentes de ambas amostras concordam com o pagamento a bloggers para a divulgação de mensagens da organização, considerando que essa é uma prática comum. Não obstante, e como visto no tabela 2, boas práticas requerem a divulgação desse pagamento. Por conseguinte e apesar do pagamento a bloggers ser uma prática não ética (Jensen, 2011), os RP de Portugal e do Brasil inquiridos tendem a perceber esta prática como aceitável, lançando dúvidas sobre a sua capacidade e autoridade como "guardiões éticos" (L'Étang, 2004). Comparando com os resultados de Toledano e Avidar (2016), os profissionais neozelandeses respondentes tenderam a rejeitar esta prática o que, uma vez mais, aproxima os nossos resultados dos profissionais israelitas inquiridos pelas colegas.

Quanto ao pagamento a especialistas em media sociais para distribuírem rumores e mensagens negativas sobre a concorrência, os dois grupos inquiridos são firmes na rejeição desta prática não ética, revelando respeito pelo mercado e consideração pela veracidade. No entanto, é importante salientar que esta é uma das afirmações do estudo mais propensa ao enviesamento "da resposta desejável".

Tabela 04: Resultados sobre a veracidade e o respeito pelo mercado

\begin{tabular}{|c|c|c|c|c|c|c|c|}
\hline Afirmações & Brasil & Brasil & Brasil & $\begin{array}{c}\text { Portu- } \\
\text { gal }\end{array}$ & $\begin{array}{c}\text { Portu- } \\
\text { gal }\end{array}$ & $\begin{array}{c}\text { Portu- } \\
\text { gal }\end{array}$ & Teste T \\
\hline
\end{tabular}




\begin{tabular}{|l|c|c|c|c|c|c|c|}
\hline & $\mathbf{N b}$ & $\boldsymbol{\mu}$ & $\mathbf{D P}$ & $\mathbf{N p t}$ & $\boldsymbol{\mu}$ & $\mathbf{D P}$ & \\
\hline $\begin{array}{l}\text { Não há problema } \\
\text { de pagar a blog- } \\
\text { gers para divulgar } \\
\text { mensagens sobre a } \\
\text { organização ou um } \\
\text { cliente. }\end{array}$ & 44 & 3,23 & 1,26 & 100 & 3,25 & 1,21 & $\begin{array}{c}\mathrm{t}(80)=1,99, \\
\mathrm{p}>0,9\end{array}$ \\
\hline $\begin{array}{l}\text { Não há problema } \\
\text { em pagar a especial- } \\
\text { istas em media so- } \\
\text { ciais para distribuir } \\
\text { rumores e men- } \\
\text { sagens negativas so- } \\
\text { bre a concorrência/ } \\
\text { oposição do meu } \\
\text { empregador ou cli- } \\
\text { ente. }\end{array}$ & 1,36 & 0,65 & 100 & 1,52 & 0,85 & $\begin{array}{c}\mathrm{t}(106)=1,98, \\
\mathrm{p}>0,2\end{array}$ \\
\hline
\end{tabular}

\section{DISCUSSÃO E CONCLUSÕES}

O comportamento ético é uma condição necessária ao estabelecimento de relações de confiança e deve acompanhar todas as atividades da organização, incluindo a presença das organizações em ambiente digital e o uso dos media sociais (Gilpin, 2010). Existem vários princípios éticos (conforme explorado na revisão de literatura) e este artigo focou-se nos eixos: da transparência associada à informação sobre a identidade, o interesse e benefício; da autenticidade das vozes evitando a dissimulação e o respeito pelos públicos e pelo mercado (Jensen, 2011). Estes eixos foram analisados enquadrados em referentes culturais onde existem diferentes níveis de transparência, liberdades humanas e uso da internet. Enquanto os portugueses têm níveis elevados de familiaridade com os media sociais; os brasileiros ainda convivem com fatores de exclusão económica, demográfica e educativa em termos de utilização da internet. Existindo, contudo, uma evolução positiva significativa nos últimos três anos.

Apesar dos diferentes desempenhos em índices internacionais, a diferença não é significativa (com exceção do Index of Human Freedom) verificando-se que o desempenho do Brasil é sempre inferior ao de Portugal. Além disso, os dois países são próximos em termos de "distância de poder" (Portugal: 69; Brasil: 63), uma das dimensões culturais de Hofstede (2001), fundamental em termos éticos, pois a aceitação da desigualdade hierárqui- 
ca revela "fechamento" democrático.

A comparação destes contextos culturais com os de Toledano e Avidar (2016), permite a utilização da Nova Zelândia como benchmark, pois este país tem melhores desempenhos nos níveis internacionais (\#1 no Fraser Institute Index of Human Freedom 2012/2013; \#2 no Corruption Perceptions Index of Transparency International e \#9 no 2014 World Press Freedom Index).

Mas, afetarão estes diferentes contextos socioculturais a atitude do RP em relação a aspetos éticos no uso dos media sociais? (QP1)

Os resultados revelam não existir diferenças significativas entre os RP de Portugal e do Brasil. De uma perspetiva normativa, é possível concluir que os RP inquiridos se consideram "guardiões éticos" no uso dos media sociais pela organização. Os inquiridos assumem que estes meios podem ajudar a aumentar o reconhecimento social e organizacional da profissão. Assim sendo, os RP são responsáveis pelos conteúdos dos media sociais e devem ser responsáveis pelas práticas comunicativas da organização em ambiente digital, dando conselhos éticos "reguladores" destas práticas.

Considerando os resultados obtidos, alinhamos as nossas conclusões com as de Toledano e Avidar (2016) reportando que práticas menos éticas - numa base atitudinal - são tendencialmente mais aceites em países com maiores níveis de corrupção percebida. Isto atendendo a que os nossos resultados se aproximam mais das respostas obtidas junto dos RP de Israel. Os próprios contextos culturais de Portugal e Brasil são mais próximos dos de Israel que da Nova Zelândia.

O nosso estudo não revela diferenças na perceção das duas amostras inquiridas sobre o conhecimento e respeito pelos princípios éticos, o que se pode relacionar com a própria profissão. Como mencionado por Gonçalves (2012), os RP de países latinos são associados à propaganda e à manipulação da opinião pública. A influência pessoal e a comunicação unidirecional são os modelos prevalecentes nestes países. Assim sendo, seguimos a hesitação de L'Étang $(2004,2011)$ sobre a legitimidade destes profissionais como "guardiões éticos" atendendo a que podem não ter capacidade nem a autoridade para fazer julgamentos sobre o que é e não é ético.

Tal é aliás revelado nas atitudes dos respondentes quanto ao eixo ético da autenticidade (QP2). A importância da autenticidade causa dúvidas aos RP brasileiros e divide opiniões entre os portugueses, atendendo a que a produção de conteúdos em nome de terceiros (líderes, figuras públicas, marca, etc.) se revela uma atividade lucrativa e promocional com níveis de 
retorno elevados (Motion et al., 2016). Da mesma forma, a transparência não é veementemente defendida pelas amostras inquiridas. Provavelmente, por recearem perder o controlo da informação, o interesse incerto do conteúdo ou o debate público sobre a organização, situações que os media sociais podem promover (Motion et al., 2016, pp. 6-7) ou pela necessidade de manter em segredo informação sobre o negócio.

Várias limitações podem ser identificadas neste estudo. Em primeiro lugar, a exiguidade das amostras respondentes (igual limitação tiveram aliás Toledano e Avidar, 2016) sendo notória a fraca participação dos profissionais em estudos académicos. Assinalamos contudo que tal também pode ser motivado pelo tema em estudo, uma vez que a ética se associa à prática profissional e pode comprometer os profissionais, sendo igualmente um tema propício ao enviesamento das respostas para a "resposta desejável". Em segundo lugar, consideramos que o estudo poderia ser enriquecido com uma abordagem qualitativa, eventualmente com a realização de entrevistas individuais e/ou coletivas com os profissionais.

\section{REFERÊNCIAS}

ABRACOM (s/d). Apresentação. Retirado de http://www1.abracom.org.br/cms/opencms/ abracom/pt/quemsomos/index.html

Bowen, S. (2007). Ethics and Public Relations. Institute for Public Relations. Retirado de http://www.instituteforpr.org/ethics-and-public-relations/ (4 de abril de 2016)

Bowen, S. A. (2013). Using classical social media cases to distil ethical guidelines for digital engagement. Journals of Mass Media Ethics: Exploring Questions of Media Morality, 28(2), 119-133. doi: http://dx.doi.org/10.1080/08900523.2013.793523

Bowen, S. A. (2013b). Ethics of public relations. In R. L. Heath (Ed.), Encyclopaedia of public relations, Vol. 1 (pp. 304-306) (2. ${ }^{a}$ Ed.). Thousand Oaks, CA: Sage.

Bowen, S. A., Hung-Baesecke, C-J. F., \& Chen, Y-R. R. (2016). Ethics as a precursor to organisation-public relationships: Building trust before and during the OPR model. Cogent Social Sciences, 2, 1-19. doi: https://doi.org/10.1080/23311886.2016.1141467

Brown, R. (2009). Public relations and the social web: using social media and web 2.0 in communications. London/Philadelphia: Kogan Page.

Cheney, G., Munshi, D., May, S., \& Ortiz, E. (2011). Encountering Communication Ethics in the Contemporary World. Principles, People, and Contexts. In G. Cheney, S. May \& D. Munshi, The Handbook of Communication Ethics (pp. 1-11). New York: Routledge.

Diga, M., \& Kelleher, T. (2009). Social media use, perceptions of decision-making power, and public relations roles. Public Relations Review, 35(4), 440-442. doi: http://dx.doi. org/10.1016/j.pubrev.2009.07.003 
CADERNOS DE COMUNICAÇÃO

UNIVERSIDADE FEDERAL DE SANTA MARIA

Duhé, S. (2015). An overview of new media research in public relations journals from 1981 to 2014. Public Relations Review, 41(2), 153-169. doi: http://dx.doi.org/10.1016/j.pubrev.2014.11.002

Einwiller, S. A., \& Steilen, S. (2015). Handling compaints on social network sites - an analysis of complaints and compaint responses on Facebook and Twitter pages of large US companies. Public Relations Review, 41(2), 195-204. doi: http://dx.doi.org/10.1016/j. pubrev.2014.11.012

Ess, C. (2011). Ethical Dimensions of New Technology/Media. In G. Cheney, S. May \& D. Munshi, The Handbook of Communication Ethics (pp. 204-220). New York: Routledge.

Fraser Institute (2013). Towards a Worldwide Index of Human Freedom. Acedido em: https://www.fraserinstitute.org/research/towards-a-worldwide-index-of-human-freedom (10 de dezembro de 2015)

Fuchs, C. (2014). Social Media: a critical introduction. Los Angeles / London: Sage.

Gilpin, D. (2010). Organisational Image Construction in a Fragmented Online Media Environment. Journal of Public Relations Research, 22(3), 265-287. doi: http://dx.doi. org/10.1080/10627261003614393

GlobalWeblndex (2014). Penetration of leading social networks in Brasil as of 4th quarter 2014. Retirado de: http://www.statista.com/statistics/284424/Brasil-social-network-penetration/ (10 de dezembro de 2015)

Goldstraw, D. (2015). Can PR Practitioners Build Positive Journalist Relationships via Social Media? In E. Ordeix, V. Carayol, \& R. Tench (Edits.), Public Relations, Values and Cultural Identity (pp. 339-359). Brussels: Peter Lang.

Gonçalves, G. (2007). Ética das relações públicas. A falta de responsabilidade social nos Códigos Éticos de Relações Públicas. Ethics of society. Ethics of Communication (pp. 1-25). Paris: IX International Association for Media and Communication Research (IAMCR) Congress.

Gonçalves, G. (2012). A identidade e a imagem das relações públicas em Portugal (The identity and image of Public Relations in Portugal). IBER: International Business and Economics Review, 3, 198-207.

Grunig, J. E. (edit) (1992). Excellence in Public Relations and Communication Management. New Jersey: Lawrence Erlbaum Associates.

Grunig, J. E., \& Grunig, L. A. (1992). Models of Public Relations and Communication. In J. E. Grunig (edit), Excellence in Public Relations and Communication Management (pp. 285-326). New Jersey: Lawrence Erlbaum Associates.

Heath, R. L. (2006). Onward into more fog: Thoughts on Public Relations' Research Directions. Journal of Public Relations Research, 18(2), 93-114.

Hofstede, G. H. (2001). Culture's Consequences. Comparing Values, Behaviours, Institutions and Organisations across Nations (2nd ed). Thousand Oaks/CA: Sage Publications.

Hyde, M. J. (2011). Ethics, Rhetoric, and Discourse. In G. Cheney, S. May \& D. Munshi, The Handbook of Communication Ethics (pp. 31-44). New York: Routledge. 
CADERNOS DE COMUNICAÇÃO

UNIVERSIDADE FEDERAL DE SANTA MARIA

Internet World Stats (2015). Internet use in Europe. Acedido em: http://www.internetworldstats.com/stats4.htm\#europe (6 de novembro de 2015)

Jensen, R. (2011). Blogala, sponsored posts, and the ethics of blogging. In B. E. Drushel \& K. German (Eds.), The ethics of emerging media: Information, social norms, and new media technologies (pp. 213-229). New York: Continuum.

Kaplan, A. M., \& Haenlein, M. (2010). Users of the world, unite! The challenges and opportunities of Social Media. Business Horizons, 53, 59-68. doi: http://dx.doi.org/10.1016/j.bushor.2009.09.003

Kent, M. L. (2008). Critical analysis of blogging in public relations. Public Relations Review, 34, 32-40. doi: http://dx.doi.org/10.1016/j.pubrev.2007.12.001

Kent, M. L., \& Taylor, M. (1998). Building Dialogic Relationships Through the World Wide Web. Public Relations Review, 24(3), 321-334. doi:10.1016/S0363-8111(99)80143-X

Kent, M. L., \& Taylor, M. (2002). Toward a dialogic theory of public relations. Public Relations Review, 28(1), 21-37. doi: http://dx.doi.org/10.1016/S0363-8111(02)00108-X

Kent, M. L., Taylor, M., \& White, W. (2003). The relationship between website design and organisational responsiveness to stakeholders. Public Relations Review, 29(1), 66-77. doi: http://dx.doi.org/10.1016/S0363-8111(02)00194-7

Kietzmann, J. H., Hermkens, K., McCarthy, I. P., \& Silvestre, B. S. (2011). Social media? Get serious! Understanding the functional building blocks of social media. Business Horizons, 54, 241-251. doi: http://dx.doi.org/10.1016/j.bushor.2011.01.005

Kim, S., Park, J.-H., \& Wertz, E. K. (2010). Expectation gaps between stakeholders and web-based corporate public relations efforts: focusing on Fortune 500 corporate websites. Public Relations Review, 36, 215-221. doi: http://dx.doi.org/10.1016/j.pubrev.2010.04.001

Lahav, T., \& Roth-Cohen, O. (2016). The changing blogosphere and its impact on public relations practice and professional ethics: The Israeli case. Public Relations Review. Advance online publication. doi: http://dx.doi.org/10.1016/j.pubrev.2016.08.006

L'Étang, J. (2004). The myth of the "ethical guardian": An examination of its origins, potency and illusions. Journal of Communication Management, 8(1), 53-67. doi: http:// dx.doi.org/10.1108/13632540410807547

L'Étang, J. (2011). Public Relations and Marketing. Ethical issues and professional practice in society. In G. Cheney, S. May \& D. Munshi, The Handbook of Communication Ethics (pp. 221-240). New York: Routledge.

Macnamara, J., \& Zerfass, A. (2012). Social media communication in organisations: The challenges of balancing openness, strategy, and management. International Journal of Strategic Communication, 6, 287-308. doi: http://dx.doi.org/10.1080/1553118X.2012.711402

Marktest. (2015). Os Portugueses e as Redes Sociais. Lisboa: Marktest Consulting.

McAllister-Spooner, S. M. (2009). Fulfilling the dialogic promise: a ten year reflective survey on dialogic internet principles. Public Relations Review, 35(3), 320-322. doi: http:// dx.doi.org/10.1016/j.pubrev.2009.03.008 
CADERNOS DE COMUNICAÇÃO

UNIVERSIDADE FEDERAL DE SANTA MARIA

Men, L. R., \& Tsai, W.-H. S. (2012). How companies cultivate relationships with publics on social network sites: evidence from China and the United States. Public Relations Review, 38, 723-730. doi: http://dx.doi.org/10.1016/j.pubrev.2011.10.006

Moloney, K. (2000). Rethinking Public Relations: PR, propaganda and democracy (2. ${ }^{a}$ ed.). London: Routledge.

Motion, J., Heath, R. L., \& Leitch, S. (2016). Social Media and Public Relations. Fake friends and powerful publics. Oxon/New York: Routledge.

Osch, W. v., \& Coursaris, C. K. (2014). Social media research: An assessment of the domain's productivity and intellectual evolution. Communication Monographs, 81(3), 1-25. doi: http://dx.doi.org/10.1080/03637751.2014.921720

Osch, W. v., \& Coursaris, C. K. (2015). A Meta-Analysis of Theories and Topics in Social Media Research. 48th Hawaii International Conference on System Sciences, 1668 - 1675. doi: http://dx.doi.org/10.1109/HICSS.2015.201

Pesquisa Brasileira de Mídia (2015). Hábitos de consumo de mídia pela população Brasileira. Acedido em: http://www.secom.gov.br/ (10 de dezembro de 2015)

PewGlobal (2014). Online Activities in Emerging and Developing Nations. Acedido em: http://www.pewglobal.org/2015/03/19/2-online-activities-in-emerging-and-developing-nations/ (4 de abril de 2016)

Phillips, D., \& Young, P. (2009). Online public relations (2. ${ }^{\mathrm{a}}$ ed.). London, UK: Kogan Page CIPR.

Reporters Without Borders (2015). 2015 World Press Freedom Index. Acedido em: http:// index.rsf.org/\#!/ (4 de abril de 2016)

Rogers, E. M. (1962/2003). Diffusion of innovations. (5 ed). New York: Free Press.

Sebastião, S. P., \& Azevedo, C. (2014). Associativismo e Ordem dos Consultores de Comunicação: vantagens e debilidades. Revista Internacional de Relaciones Públicas, 4(7), 39-52. Retirado de: http://revistarelacionespublicas.uma.es/index.php/revrrpp/article/ view/256 (4 de abril de 2016)

Seltzer, T., \& Mitrook, M. A. (2007). The dialogic potential of weblogs in relationship building. Public Relations Review, 33(2), 227-229. doi: http://dx.doi.org/10.1016/j.pubrev.2007.02.011

Sriramesh, K., \& Verčič, D. (Eds.). (2009). The global public relations handbook: Theory, research and practice (ed. Rev.). New York: Routledge.

Tench, R., Zerfass, A., Verhoeven, P., Vercic, D., Moreno, A., \& Okay, A. (2013). Communication Management Competencies for European Practitioners. Acedido em European Communication Professionals Skills and Innovation Programme (ECOPSI): http://www. ecopsi.org.uk/ (4 de abril de 2016)

Toledano, M., \& Avidar, R. (2016). Public relations, ethics, and social media: A cross-national study of PR practitioners. Public Relations Review, 42(1), 161-169. doi: http://dx.doi. org/10.1016/j.pubrev.2015.11.012

Toledano, M., \& Wolland, L. (2011). Ethics 2.0: Social media implications for professional 
CADERNOS DE COMUNICAÇÃO

UNIVERSIDADE FEDERAL DE SANTA MARIA

communicators. Ethical space: The international journal of communication ethics, $8(3 / 4)$, $43-52$.

Transparency International (2014). Corruption Perceptions Index. Acedido em: https:// www.transparency.org/cpi2014/infographic/compare (4 de abril de 2016)

Treem, J. W., \& Leonardi, P. M. (2012). Social media use in organisations: Exploring the affordances of visibility, editability, persistence, and association. Communication Yearbook, 36, 143-189. doi: http://dx.doi.org/10.1080/23808985.2013.11679130

Valentini, C. (2015). Is using social media "good" for the public relations profession? A critical reflection. Public Relations Review, 41(2), 170-177. doi: http://dx.doi.org/10.1016/j. pubrev.2014.11.009

Vásquez, I., \& Štumberger, T. (2012). An index of freedom in the world. In F. McMahon (Ed.), Towards a worldwide index of human freedom (pp. 55-106). Fraser Institute. Retirado de: http://www.fraserinstitute.org/uploadedFiles/fraser-ca/Content/research-news/ research/publications/towards-a-worldwide-index-of-human-freedom.pdf (4 de abril de 2016)

Vercic, D., Vercic, A. T., \& Sriramesh, K. (2014). Looking for digital in public relations. Public Relations Review, 41, 142-152. doi: http://dx.doi.org/10.1016/j.pubrev.2014.12.002

Wright, D. K. (2001). The magic communication machine: Examining the Internet's impact on public relations, journalism, and the public. Gainesville, FL: The Institute for Public Relations.

Wright, D. K., \& Hinson, M. D. (2015). Examining social and emerging media use in public relations practice: A ten-year longitudinal analysis. Public Relations Journal, 9(2), 1-26. Retirado de https://www.prsa.org/Intelligence/PRJournal/Documents/2015vogno2Wrighthinson.pdf (4 de abril de 2016)

Ye, L., \& Ki, E.-J. (2012). The status of online public relations research: An analysis of published articles in 1992-2009. Journal of Public Relations Research, 24(5), 409-434. doi: http://dx.doi.org/10.1080/1062726X.2012.723277

Young, P. M. (2015). A History of the Future: Concepts for Telling the Story of Online PR. In E. Ordeix, V. Carayol, \& R. Tench (Edits.), Public Relations, Values and Cultural Identity (pp. 31-56). Brussels: Peter Lang.

Zerfass, A., Tench, R., Vercic, D., Verhoeven, P., \& Moreno, A. (2014). European communication monitor 2014. Excellence in strategic communication - Key issues, leadership, gender and mobile media. Results of a survey in 42 countries. Brussels: EACD/EUPRERA. 


\section{Sónia Pedro Sebastião}

Doutorada em Ciências Sociais, na especialização de Ciências da Comunicação. É Professora Associada no ISCSP, Universidade de Lisboa e investigadora integrada do CAPP/FCT. A sua investigação incide principalmente sobre temas relacionados com as Relações Públicas e a Comunicação Estratégica, tendo dezenas de publicações e participações em Conferências nacionais e internacionais na área.

E-mail: ssebastiao@iscsp.ulisboa.pt

\section{Giovana Zulato}

Mestre em Comunicação Social, na vertente de Comunicação Estratégica pelo ISCSP, Universidade de Lisboa. Tem cerca de 10 anos de experiência em comunicação organizacional, já atuou como assessora de imprensa, produtora de conteúdo institucional para publicações impressas e online, community manager, entre outras funções. A sua especialidade é a Comunicação Interna, área em que atuou em agências de comunicação e em empresas (no Brasil e em Portugal).

E-mail: giovanazulato@yahoo.com.br

\section{Giovana Zulato}

Mestranda em Comunicação Social, na vertente de Comunicação Estratégica pelo ISCSP, Universidade de Lisboa e pós graduada em Comunicação Estratégica Digital na mesma instituição. Tem experiência profissional em Agências de Comunicação em Portugal, na área das relações com os media e media digitais. Integra atualmente o departamento de Comunicação Corporativa de uma grande empresa de telecomunicação em Portugal (a NOS) tendo, entre várias funções, a responsabilidade de gestão de redes sociais.

E-mail: taniabelosantos@gmail.com 